\title{
Dynamic predictors of VILI risk: beyond the driving pressure
}

\author{
John J. Marini ${ }^{1 *}$ and Samir Jaber ${ }^{2}$
}

As currently implemented, lung protective ventilation concentrates on certain static characteristics of the individual tidal cycle-tidal volume (TV), plateau pressure, PEEP, and recently, on the difference between the latter two static values, the driving pressure (DP) [1]. The rationale for focusing on any of these has been based primarily on concept, focused animal experiments, and clinical data supporting their relative importance. However, the physical process that causes ventilator-induced lung injury (VILI) has been difficult to pin down. It is clear that mechanical forces, lung patho-anatomy and non-ventilatory characteristics each contribute. Excessive stretch, strain, and tidal opening and closure may all be important, but the precise mechanism through which they act remains unclear. Dynamic characteristics-frequency, flow rate, strain rate-have recently been emphasized as key determinants of whether the 'static' variables inflict injury [2]. In this issue, Gattinoni and colleagues present an elegant and persuasive argument that energy delivered per unit time ('power') is a unifying entity into which most key ventilator settings and forces relevant to VILI can be channeled, thus providing a "composite index" by which to translate this insight into clinical practice [3]. As clearly stated by the authors themselves, the mechanical power concept in the genesis of VILI is not new. Rather, the novelty of this work lies in proposing and validating a mathematical description of machine power responsive to the relative contributions of its bedside-adjustable components (TV, frequency, $\Delta P_{\mathrm{aw}}$, PEEP, $I: E$, flow). This interesting and provocative proposal urges a potentially important conceptual shift in our thinking that deserves to be carefully examined.

\footnotetext{
*Correspondence: marin002@umn.edu

1 University of Minnesota, Minneapolis/Saint Paul, MN, USA Full author information is available at the end of the article This editorial refers to the article available at: doi:10.1007/s00134-016-4505-2
}

Ventilators regulate either pressure or flow but not both simultaneously, even in the dual-control modes available in some modern ventilators. This restriction arises because the product of developed airway pressure (force per unit area) and the resulting delivered volume (area-length product) defines the energy cost of the breath to overcome resistance $(R)$ and elastance $(1 / C)$. Inflation is subject to the energy conservation law and therefore is constrained mathematically by a relationship known as the equation of motion of the respiratory system [4]. The total inflation pressure $\left(P_{\text {tot }}\right)$ corresponding to any volume $(V)$ above the fully relaxed value (FRC) must be accounted for in the sum of dissipated and conserved pressures, usually approximated as: $P_{\text {tot }}=$ flow $\times R+V / C+$ PEEP $_{\text {tot }}$. (The energy expended during passive ventilation-the work of each tidal inflation-is the product of the proximal airway pressure and the volume change it produces [5]. Power or energy load, defined as work per unit time, takes the number of energy cycles per minute (breathing frequency, $f$ ) into account.

The proposition that delivered power relates directly to VILI has intuitive appeal. Experimental studies have shown that, although the peak magnitudes of tidal alveolar stresses and strains are very important, other factors condition the resulting damage. The excursion of tidal pressure (DP) appears to be more important than the maximum (plateau) pressure applied [1], and the frequency of potentially injurious cycling helps determine tissue damage [6]. Flow rate and profile, clinically adjustable variables often de-emphasized in the lung protective strategy, have also been shown to be influential, even when plateau and driving pressures remain constant $[2$, 7]. Whether raising PEEP proves protective or deleterious has been thought to depend on its ability to recruit new units and whether DP or plateau pressure remains unchanged during the increase.

\section{Springer}


The alluring aspects of this 'VILI-Power' hypothesis are several. In accounting for key dynamic (as well as static) variables, it lends mechanistic plausibility to the observation that DP is more influential than plateau pressure. If damage resulted from excess power, it would not depend exclusively on the maximal pressures achieved during the individual tidal cycle but rather on the entirety of the inspiratory pressure excursion (DP) and on the frequency of its application. Moreover, the power hypothesis builds upon the solid science that preceded it by integrating and assigning relative weights to machine settings which separately have been incriminated as contributors to VILI but individually cannot fully account for injury risk. What is more, these contributing components of the power equation can be quantified, ranked, and condensed into a single practical index with the potential to guide machine adjustments at the bedside.

Like most provocative and insightful work, questions regarding possible shortcomings and needed refinements come rather quickly to mind. However attractive this concept might be, it may need to be further polished before it can be relied upon to safely guide ventilation. For example, it is certainly true that the equation of motion accounts for the flows and pressures the ventilator generates relative to atmosphere and therefore determines the total work and energy that the ventilator must perform during a given inflation. But airway pressure expands both the lung and chest wall, and abnormal stiffness of the latter sometimes accounts for a sizeable fraction of the delivered pressure and work per cycle [8]. What is more, the local tissue tensions within the mechanically heterogeneous lung (and, by implication, the unmeasured regional power relevant to micro-injury) may be affected by the force amplification that occurs in zones of stress focusing [9]. This vital issue of lung heterogeneity is not readily quantified, and clinicians at the bedside are left with what we can measure-the elements of the equation of motion. Using transpulmonary pressure, however, would seem a logical and feasible option for calculating lung injury power.

There are other concerns. Even though logically weighted, not all components of the proposed power equation contribute to VILI in an obvious way. For instance, although flow magnitude and flow profile (attack rate $\mathrm{d} P / \mathrm{d} t$ ) relate to the aggressiveness of lung tissue expansion during inflation and may contribute to damage for that reason [2, 7], it is difficult to link power dissipated in proximal airway resistance directly to noxious events at the alveolar level.

Another important question relates to the inclusion of PEEP in the power computation. As nicely discussed by the authors, PEEP invariably increases global tissue stresses and strains when tidal volume remains unchanged
[3]. Such elevations of baseline pressure amplify tissue tensions and the potentially damaging effects of dynamic tidal excursions. Furthermore, PEEP adds to the pressure that the machine must generate, which clearly expends energy in doing so. But is the PEEP component of the power equation directly related to tissue injury? (Fig. 1). The block of pressure-volume area that corresponds to the ventilator's work against PEEP is temporarily stored as potential energy within the elastic tissues of the respiratory system; it later converts to kinetic energy as the gas escapes to the atmosphere across the exhalation valve.

If both resistance and PEEP components of the ventilating power calculation are sharply discounted, the damaging influence related to the key remaining term of the equation of motion, the DP term $(\mathrm{VT} / \mathrm{C})$, would assume even greater prominence than its quadratic $(\times 2)$ volume exponent in the energy and power equations implies. Focusing attention on delivered power supports the importance of DP itself to the VILI but emphasizes that the frequency at which such excursions is repeated (and minute ventilation $V_{\mathrm{E}}$ \} helps determine the true hazard. Rather than the raw driving pressure $(\mathrm{DP}=\mathrm{VT} / C)$ that currently is favored, a better indicator of injury risk for clinical purposes might be the "driving power" (if compliance adjusted for the reduced capacity of the 'baby lung'): Driving Power $=[f \times \mathrm{VT} \times \mathrm{DP}] / 10 \mathrm{C}=\left[\mathrm{DP} \times V_{\mathrm{E}}\right] / 10 \mathrm{C}$.

As the authors emphasize, VILI derives from the interaction between the causal factors of two broad categories (lung conditions and machine output). For example, the same mechanical power may kill a rat but cause no harm to the much larger pig. Obviously, to gauge risk among our patients the mechanical power must be first be normalized, at least for aerated lung capacity. (For a given individual, however, tracking risk with total power would still seem prudent during machine adjustments.) The present paper [3] validates an analytical approach to quantifying the raw power load. For humans, dangerous levels (if they exist) must be defined. Finally, even though the raw value of calculated power delivery may be high, tissue damage might be inconsequential unless specific thresholds of trans-alveolar pressure and tissue strain per cycle are crossed. In other words, the pressure range over which power is delivered may be critical to outcome. An attractive possibility to tie the existence of a pressure range to an injury threshold is that local stress risers amplify the actual effect of applied pressure on local tissue tension-and locally inflicted power-that results from a given transpulmonary driving pressure. Damage would then propagate from those zones [10].

As already noted, most power delivered to the lung is stored temporarily in the respiratory system during inflation and then released during exhalation. However, some power is expended within the lung itself in reversibly 

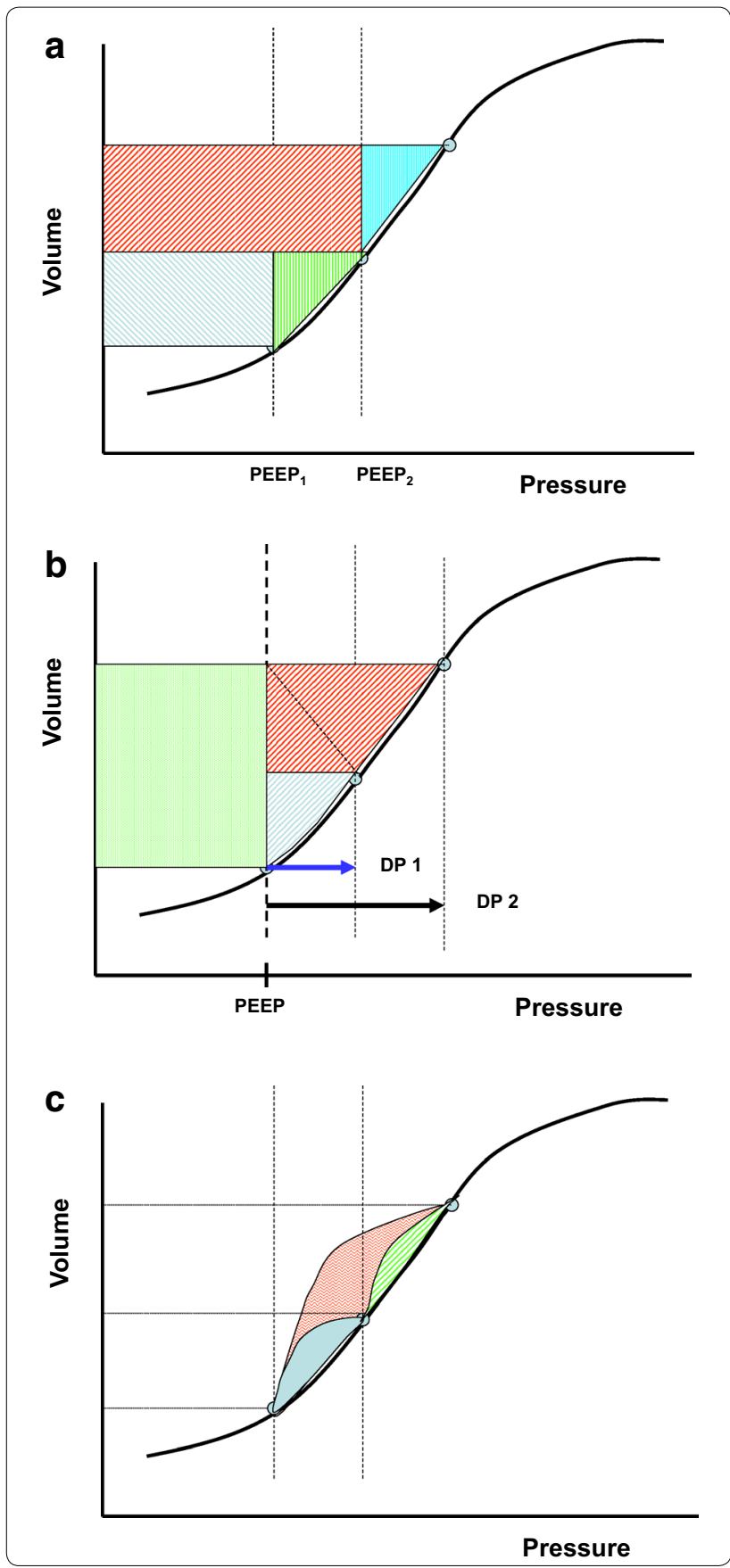

deforming tissue, overcoming viscoelastance and surface tension, recruiting unstable units, displacing blood, etc. Perhaps the product of cycling frequency and the hysteresis area enclosed within the tidal trans-pulmonary pressure-volume loop-a PEEP and recruitment-influenced variable that quantifies the lung-dissipated energy per cycle-may enhance and refine the power principle in the attempt to home in on the true proximate mechanical cause of VILI.
Fig. 1 Relationships of PEEP and driving pressure (DP) to energy imparted to the passive tissues of the respiratory system during a single inflation. (Flow resistive work performed by the machine is not illustrated.) Colored areas (pressure $\times$ volume) represent mechanical work. a For a given tidal volume and DP, an increase of PEEP (from PEEP1 to PEEP2) increases inspiratory storage of energy (rectangles) while delivering similar incremental (dynamic) energy (triangles). The PEEP-related energy stored during inflation, along with its dynamic counterpart, is then released during exhalation as it dissipates across the resistance of the circuit and exhalation valve, allowing airway pressure to return to the PEEP level. $\mathbf{b}$ A doubling of tidal volume and DP from the same PEEP level raises dynamic energy disproportionately to the tidal volume and driving pressure. c During deflation, some energy is retained by the lung as the remainder dissipates across the airways, circuitry and exhalation valve. Lenticular-shaped areas quantify the energy retained at two PEEP levels with the same DP (blue and green) as well as the energy retained by doubling the tidal volume with the same PEEP

The integrating concept of mechanical power represents a significant conceptual step forward in our understanding of how to piece together the experimental and clinical information related to safe and effective lung ventilation. By emphasizing the 'ergotrauma' of delivered power, this important work [3] calls attention to the often neglected mandate to reduce the demand for ventilation as well as the dynamic stress imposed by each individual tidal breath. The innovative concepts elaborated in this impressive work will advance future efforts to refine lung protective strategies for ventilation.

\section{Author details \\ ${ }^{1}$ University of Minnesota, Minneapolis/Saint Paul, MN, USA. ${ }^{2}$ University of Montpellier, Montpellier, France.}

\section{Compliance with ethical standards}

\section{Conflicts of interest}

John J. Marini and Samir Jaber declare no professional, academic, competitive or financial conflict of interest.

Received: 28 August 2016 Accepted: 29 August 2016

Published online: 16 September 2016

\section{References}

1. Amato MB, Meade MO, Slutsky AS, Brochard L, Costa EL, Schoenfeld DA, Stewart TE, Briel M, Talmor D, Mercat A, Richard JC, Carvalho CR, Brower RG (2015) Driving pressure and survival in the acute respiratory distress syndrome. N Engl J Med 372(8):747-755

2. Protti A, Maraffi T, Milesi M, Votta E, Santini A, Pugni P, Andreis DT, Nicosia F, Zannin E, Gatti S, Vaira V, Ferrero S, Gattinoni L (2016) Role of strain rate in the pathogenesis of ventilator-induced lung edema. Crit Care Med 44(9):e838-e845

3. Gattinoni L, Tonetti T, Cressoni M, Cadringher P, Herrmann P, Mörer O, Protti A, Gotti M, Chiurazzi C, Carlesso E, Quintel M (2016) Ventilatorrelated causes of lung injury: the mechanical power. Int Care Med (This Issue) 
4. Otis AB, Fenn WO, Rahn H (1950) Mechanics of breathing in man. J Appl Physiol 11:592-607

5. Marini JJ, Rodriguez RM, Lamb VJ (1986) Bedside estimation of the inspiratory work of breathing during mechanical ventilation. Chest 89(1):56-63

6. Hotchkiss JR, Blanch LL, Murias G, Adams AB, Olson D, Wangensteen OD, Leo PH, Marini JJ (2000) Effects of decreased respiratory frequency on ventilator induced lung injury. Am J Respir Crit Care Med 161:463-468

7. Maeda Y, Fujino Y, Uchiyama A, Matsuura N, Mashimo T, Nishimura M (2004) Effects of peak inspiratory flow on development of ventilatorinduced lung injury in rabbits. Anesthesiology 101 (3):722-728

8. Cortes-Puentes GA, Keenan JC, Adams AB, Parker ED, Dries DJ, Marini JJ (2015) Impact of chest wall modifications and lung Injury on the correspondence between airway and transpulmonary driving pressures. Crit Care Med 43(8):e287-e295
9. Cressoni M, Cadringher P, Chiurazzi C, Amini M, Gallazzi E, Marino A, Brioni M, Carlesso E, Chiumello D, Quintel M, Bugedo G, Gattinoni L (2014) Lung inhomogeneity in patients with acute respiratory distress syndrome. Am J Respir Crit Care Med 189(2):149-158. doi:10.1164/rccm.201308-1567OC

10. Cressoni M, Chiurazzi C, Gotti M, Amini M, Brioni M, Algieri I, Cammaroto A, Rovati C, Massari D, di Castiglione CB, Nikolla K, Montaruli C, Lazzerini M, Dondossola D, Colombo A, Gatti S, Valerio V, Gagliano N, Carlesso E, Gattinoni L (2015) Lung inhomogeneities and time course of ventilatorinduced mechanical injuries. Anesthesiology 123(3):618-627 